10 years ESJ

Special edition

\title{
Reinvención de las PYMES con el uso de Herramientas y Estrategias de Mercadotecnia ante la Pandemia del COVID-19
}

\author{
Lida Zoraida Jiménez Calixto \\ María Gabriela Pérez Ramos
}

Universidad Tecnológica de Huejotzingo, Puebla, México

Doi:10.19044/esj.2021.v17n4p61

Submitted: 28 October 2020

Accepted: 29 November 2020

Published: 07 February 2021
Copyright 2021 Author(s)

Under Creative Commons BY-NC-ND

4.0 OPEN ACCESS

Cite As:

Jiménez Calixto L.Z. \& Pérez Ramos M.G. (2021). Reinvención de las PYMES con el uso de Herramientas y Estrategias de Mercadotecnia ante la Pandemia del COVID-19. European Scientific Journal, ESJ, 17(4), 61. https://doi.org/10.19044/esj.2021.v17n4p61

\section{Resumen}

Durante el proceso de estadía práctica en el sector empresarial, que llevaron a cabo los alumnos de la carrera: de Ingeniería en Desarrollo Empresarial (IDE) en el periodo enero - abril 2020 y los de Técnico Superior Universitario en Desarrollo de Negocios área Mercadotecnia (DNM), en el período mayo-agosto 2020, de la Universidad Tecnológica de Huejotzingo (UTH), se recuperaron las experiencias del trabajo que se realizó con las PyMEs ubicadas en diferentes lugares del estado de Puebla en México, que abarcó dos etapas, la primera que coincide con el inicio del confinamiento, y una segunda fase que abarca el periodo donde se llevó a cabo el cierre total de los negocios, por lo que, se considera que a partir de la pandemia las PyMEs tuvieron que reinventarse a través de la implementación de nuevas estrategias y herramientas que para el caso que se analiza se enfoca en el ámbito de la mercadotecnia. Específicamente los casos que se analizaron, tienen que ver con un proceso de cambio y adaptación de las empresas que emigraron del comercio tradicional, al uso e incorporación de procesos desconocidos por decirlo de alguna manera, y que les permitió seguir a flote con sus ventas durante la pandemia. Para algunas empresas es una realidad el que por primera vez aplicaron herramientas mercadológicas que en ese momento fueron totalmente nuevas, esa transformación les permitió continuar a pesar de las circunstancias y la 
incertidumbre. Los beneficios alcanzados se muestran en los resultados y giran en torno de cómo se implementó el uso de las herramientas y estrategias para cada negocio, que van desde quienes hicieron uso de las redes sociales como WhatsApp, Facebook, hasta los que implementaron nuevas formas de pago por medios electrónicos, el uso de envío por mercado libre, así como los que se decidieron por el comercio electrónico. Finalmente, se muestra como los propietarios, rompieron paradigmas al aceptar la implementación de los cambios, queda demostrado que la reinvención de las PyMEs es posible aún en tiempos de pandemia.

Palabras clave: Pymes, herramientas, estrategias de mercadotecnia, promoción de ventas

\title{
Reinventing SMEs Through the use of Marketing Tools and Strategies to Face The COVID-19 Pandemic
}

\author{
Lida Zoraida Jiménez Calixto \\ María Gabriela Pérez Ramos
}

Universidad Tecnológica de Huejotzingo, Puebla, México

\begin{abstract}
The lockdown affected the business sector, students of Business Development Engineering (IDE) in the period between January - April 2020, and those of Higher University Technician in Business Development Marketing area (DNM) in the period May-August 2020 at the Technological University of Huejotzingo (UTH). The experiences of the work that was carried out with SMEs located in different places of the state of Puebla in Mexico covered two stages: the first stage coincides with the beginning of the confinement, while the second stage covers the period where the total closure of the businesses took place. It is considered that from the time the pandemic started, the SMEs had to reinvent themselves through the implementation of new strategies and tools which focuses on the field of marketing. Specifically, the cases that were analyzed have to do with a process of change and adaptation of companies that emigrated from traditional commerce to the use and incorporation of unknown processes. It also allowed them to stay afloat with their sales during the pandemic. For some companies, it is a reality that for the first time, they applied marketing tools that were totally new at that time. This transformation allowed them to continue despite the circumstances and uncertainty. The benefits achieved are shown in the results, and it revolves around how the use of the tools and strategies was implemented for each business. This ranges from those who made use of social networks such as WhatsApp and Facebook to those who
\end{abstract}


implemented new forms of payment by electronic means, or the use of free market shipping, as well as those who decided on electronic commerce. Finally, it shows how the owners broke paradigms by accepting the implementation of the changes. It has therefore been demonstrated that the reinvention of SMEs is possible even in times of pandemic.

Keyword: SMEs, tools, marketing strategies, sales promotion

\section{Introducción}

El mundo cambio repentinamente al verse afectado por la pandemia, a raíz de la propagación del COVID-19, lo que detonó en el confinamiento, y en el ámbito empresarial, esta situación ha ocasionado fuertes estragos en las pequeñas y medianas industrias en México, obligándolas a buscar nuevas oportunidades al hacer uso de la digitalización, en la exploración de herramientas que faciliten los procesos de compra, distribución, formas de pago, desarrollo de portales, entre otras más, con la finalidad de mantenerse a flote para seguir vigentes en el mercado.

Según los datos de la Secretaría de Trabajo, el 70\% de las PyMEs en México seguirán laborando a distancia o "desde casa", aún después de finalizada la contingencia, por lo que se convierte en una necesidad contar con herramientas tecnológicas que brinden un procesamiento inmediato, para llevar a cabo transacciones seguras, por lo tanto, la incorporación de nuevas herramientas se volvió indispensable para los negocios.

Sin duda la adaptabilidad, transformación y digitalización han sido las respuestas más constantes y asertivas por parte de las pequeñas y medianas empresas (PyMEs) de México durante la llamada "nueva normalidad", en el trabajo que se presenta se muestra como cinco empresas de diversos giros y tamaños, ubicadas en diferentes lugares del estado de Puebla, al verse obligadas a cerrar sus instalaciones, deciden buscar alternativas para mantenerse en operaciones.

En la elección de las herramientas había que analizar las características, tamaño, recursos, de cada negocio, para que una vez diseñadas las estrategias, presentarlas con los responsables de cada negocio para su aprobación y puesta en marcha, lo que llevo al planteamiento de la siguiente pregunta ¿Cuáles son las estrategias y herramientas de mercadotecnia, que permitirán la reinvención de las Pymes para seguir vigentes en el mercado a raíz de la pandemia ocasionada por el COVID-19? a su vez, para para dar dirección al trabajo se estableció el objetivo que fue el punto de partida en el inicio de la investigación. 


\section{Objetivo del proyecto}

Implementar estrategias y herramientas de mercadotecnia en las empresas, con el apoyo de los alumnos durante la estadía-práctica en el sector empresarial, que llevaron a cabo en el período de enero-agosto 2020, para brindar soluciones que les permitan a los negocios seguir vigentes en el mercado durante la pandemia derivada por el COVID-19.

\section{Método}

Se considera que el estudio es de corte cualitativo, de acuerdo con Jiménez (2004), en la Investigación de Marketing se utilizan técnicas enfocadas a obtener información sobre el mercado, con el fin de facilitar la toma de decisiones con el mínimo de incertidumbre y que resulten lo más acertadas posibles con el mínimo riesgo, de tal forma que la información para la realización de este trabajo se obtuvo de los proyectos realizados por los alumnos en las Mypes una vez concluida la estadía-práctica, se revisaron cada uno de los proyecto lo que permitió la identificación de las estrategias y herramientas diseñadas, cono fueron implementadas, los resultados obtenidos. La investigación es de cohorte transversal porque se realizó en un mismo período, previamente conocido y establecido, descriptiva porque se describen las acciones realizadas en cada una de la PyMES.

\section{Población de estudio}

De todos los alumnos que realizaron la estadía-practica durante el período enero-agosto 2020, el factor fundamente para seleccionar los proyectos, fue la autorización de las PyMEs para permitir a los estudiantes llevar a cabo la implementación de estrategias y herramientas de mercadotecnia, para seguir vigentes en el mercado, a pesar de la contingencia ocasionada por el COVID-19.

Es importante mencionar que las estadías abarcan un cuatrimestre, durante el período enero-abril corresponde a los estudiantes de ingeniería la realización de su estadía-práctica, fueron ellos los que se encontraban en las empresas de forma presencial hasta el día 20 de marzo que se declaró oficialmente el cierre de escuelas y negocios, lo que llevó a que el periodo restante lo concluyeron desde casa de manera virtual.

En el cuatrimestre mayo-agosto, les corresponde a los alumnos del TSU la realización de la estadía, por lo que todos los estudiantes trabajaron los proyectos desde casa por lo que fueron ubicados en las empresas que aceptaron la realización de proyectos bajo estas condiciones, de tal forma que fueron tres los propietarios que aceptaron la propuesta para incorporar estrategias de mercadotecnia para reinventarse y continuar vigentes durante la Pandemia. 
Las variables consideradas para la descripción de los hallazgos encontrados fueron dos:

1. Tamaño de la empresa

2. Área de oportunidad

\section{Materiales}

Proyectos finales realizados por los alumnos durante su estancia en estadía - práctica en el sector empresarial (obsérvese la tabla 1).

Tabla 1. Pymes que participaron en el estudio

\begin{tabular}{|c|c|c|c|c|c|}
\hline $\begin{array}{c}\text { Nombre de la } \\
\text { empresa o } \\
\text { Negocio }\end{array}$ & $\begin{array}{l}\text { Propietario(a) } \\
\text { y dirección }\end{array}$ & $\begin{array}{c}\text { Tamaño } \\
\text { de la } \\
\text { empresa }\end{array}$ & $\begin{array}{c}\text { periodo } \\
\text { de } \\
\text { estadía }\end{array}$ & Actividad & $\begin{array}{c}\text { Área de } \\
\text { oportunidad }\end{array}$ \\
\hline $\begin{array}{c}\text { 1.CECSAMEX } \\
\text { (Tlaxcalancingo) }\end{array}$ & Anónimo & mediana & $\begin{array}{c}\text { Enero- } \\
\text { abril }\end{array}$ & $\begin{array}{l}\text { Producción y } \\
\text { venta de } \\
\text { materiales } \\
\text { para la } \\
\text { industria del } \\
\text { campo y la } \\
\text { construcción. }\end{array}$ & $\begin{array}{c}\text { Proyecto } \\
\text { inicial rediseño } \\
\text { de la página } \\
\text { Web, al } \\
\text { anunciarse la } \\
\text { pandemia } \\
\text { cambio a e- } \\
\text { comerce. }\end{array}$ \\
\hline $\begin{array}{l}\text { 2.CECYTE } \\
\text { (Cholula) }\end{array}$ & $\begin{array}{l}\text { Lic. Roberto } \\
\text { A. Velázquez } \\
\text { Tlaseca } \\
\text { (director del } \\
\text { Plantel) }\end{array}$ & mediana & $\begin{array}{c}\text { Enero- } \\
\text { abril }\end{array}$ & $\begin{array}{l}\text { Educación } \\
\text { media } \\
\text { superior }\end{array}$ & $\begin{array}{l}\text { Mercadotecnia } \\
\text { y relaciones } \\
\text { publicas }\end{array}$ \\
\hline $\begin{array}{l}\text { 3.PC IXOYE } \\
\text { (San Martín } \\
\text { Texmelucan) }\end{array}$ & $\begin{array}{c}\text { Antonio Silva } \\
\text { Zamudio }\end{array}$ & pequeña & $\begin{array}{l}\text { Mayo- } \\
\text { agosto }\end{array}$ & $\begin{array}{l}\text { soluciones } \\
\text { electrónicas }\end{array}$ & $\begin{array}{l}\text { Promoción de } \\
\text { ventas. }\end{array}$ \\
\hline
\end{tabular}

\section{Hallazgos}

La implementación de cambios en la diferentes PyMEs que participaron en el estudio se describe a continuación, en el orden en que aparecen en la tabla 1. En cada uno de los casos analizados, se resalta como fue el proceso que siguieron desde el diseño hasta la puesta en marcha de las herramientas y estrategias de mercadotecnia para que los negocios continuaran vigentes durante la pandemia por el COVID-19.

\section{CECSAMEX}

Es una empresa de tamaño mediano que lleva 22 años en el mercado, el proyecto fue realizado por la alumna Ana Karen Guzmán García que cursaba el $11^{\circ}$ cuatrimestre de Ingeniería, se presentó una situación peculiar 
en el desarrollo del proyecto, si bien, al inicio de la estadía-práctica la primer propuesta fue el desarrollo de una página Web para promover las ventas de productos de la empresa, una vez que la situación cambió por el COVID-19, dejó entrever los problemas que se avecinaban para marzo de 2020, llegado el momento y con el cierre de la empresa, el proyecto cambio y se optó por el desarrollo del comercio electrónico, como estrategia para que la empresa prosiguiera con las ventas en el mercado.

La idea principal se tomó a partir de lo que establece el E Business to Consumer (B2C), este tipo de comercio electrónico ha generado toda una revolución en la sociedad, ya que cada vez está más extendido e, incluso desde el móvil o cualquier otro dispositivo, permite realizar la compra de un producto en cualquier momento. Las principales ventajas que aporta este modelo de e-commerce se basan fundamentalmente en la rapidez con la que se puede realizar esa conversión y en la posibilidad de buscar y comparar precios (Guajardo, 2019).

\section{Diseño del modelo de comercio electrónico para la empresa Cecsamex}

Para el desarrollo de la idea del B2C, se realizó un mistery shopper a los sitios web de tres diferentes empresas, Liverpool, Amazon y Walmart, con la finalidad de identificar los puntos clave del contenido en sus páginas, y los factores que las han llevado al éxito en ventas por internet, la información recopilada permitió encontrar los puntos débiles y fuertes de diversos aspectos para la empresa, y que fueron la base para el diseño del modelo de CECSAMEX.

\section{Pasos para la propuesta de Cecsamex}

Paso 1. Elaboración de propuesta sobre cómo quedaría la página web.

Paso 2. Acceso: www.cecsamex.com

Paso 3. Agregar los elementos principales que debe contener toda tienda online, Cecsamex ya cuenta con algunos y a continuación mencionare los que se proponen, los cuales son los siguientes:

- Al entrar a la página inmediatamente se abrirá un chat con el siguiente mensaje:

"Buenas tardes, bienvenido a Cecsamex ¿En qué te puedo ayudar?"

- Motor de búsqueda: La idea es facilitarle al cliente poder encontrar nuestros productos y servicios, es indispensable disponer de un potente motor de búsqueda.

- Agregar una barra en la que diga, promociones, los productos más vendidos y proceso de compra.

- Proceso de compra: Es aquel que es directo y se encuentra guiado mediante mensajes de información. 
- En la esquina superior derecha, estarán los pedidos e iniciar sesión, junto al carrito de compras

- Aspectos legales: La empresa ya cuenta con todos los aspectos legales para uso de la tienda online, que incluye, términos y condiciones y aviso de privacidad.

- En la parte superior izquierda, Información corporativa (Nuestra empresa) Estará estructurada por: cronología, nosotros, misión, visión, valores.

- Distribución: Aparecerá un video que se encuentra actualmente en la página de Cecsamex y en la parte de abajo se pondrá, "Cobertura total en todo el país".

- Productos: Al darle click se abrirá una barra en la cual estarán clasificados los productos y al momento de seleccionar alguno, en automático se desglosara la gama de productos que tenga que ver con el producto seleccionado junto con su precio.

- En la parte de abajo estarán 3 apartados:

- En el primer apartado dirá Soporte técnico y estará clasificado en:

Pedidos

Facturación

Procesos de compra

- En el segundo apartado dirá acerca de Cecsamex y estará clasificado en:

¿Quiénes somos?

Bolsa de trabajo

- En el tercer apartado dirá "Contáctanos" y estará clasificado en:

Número de teléfono

Correo electrónico

- Al final de la página estarán:

Términos y condiciones

Aviso de privacidad

Iconos de las redes sociales que maneja Cecsamex

\section{Implementación de la página en la empresa CECSAMEX}

Una vez aprobados los contenidos, se hicieron las pruebas y modificaciones correspondientes para poner en marcha las ventas por internet de la empresa y finalmente el boceto de la página quedó como se muestra a continuación. 


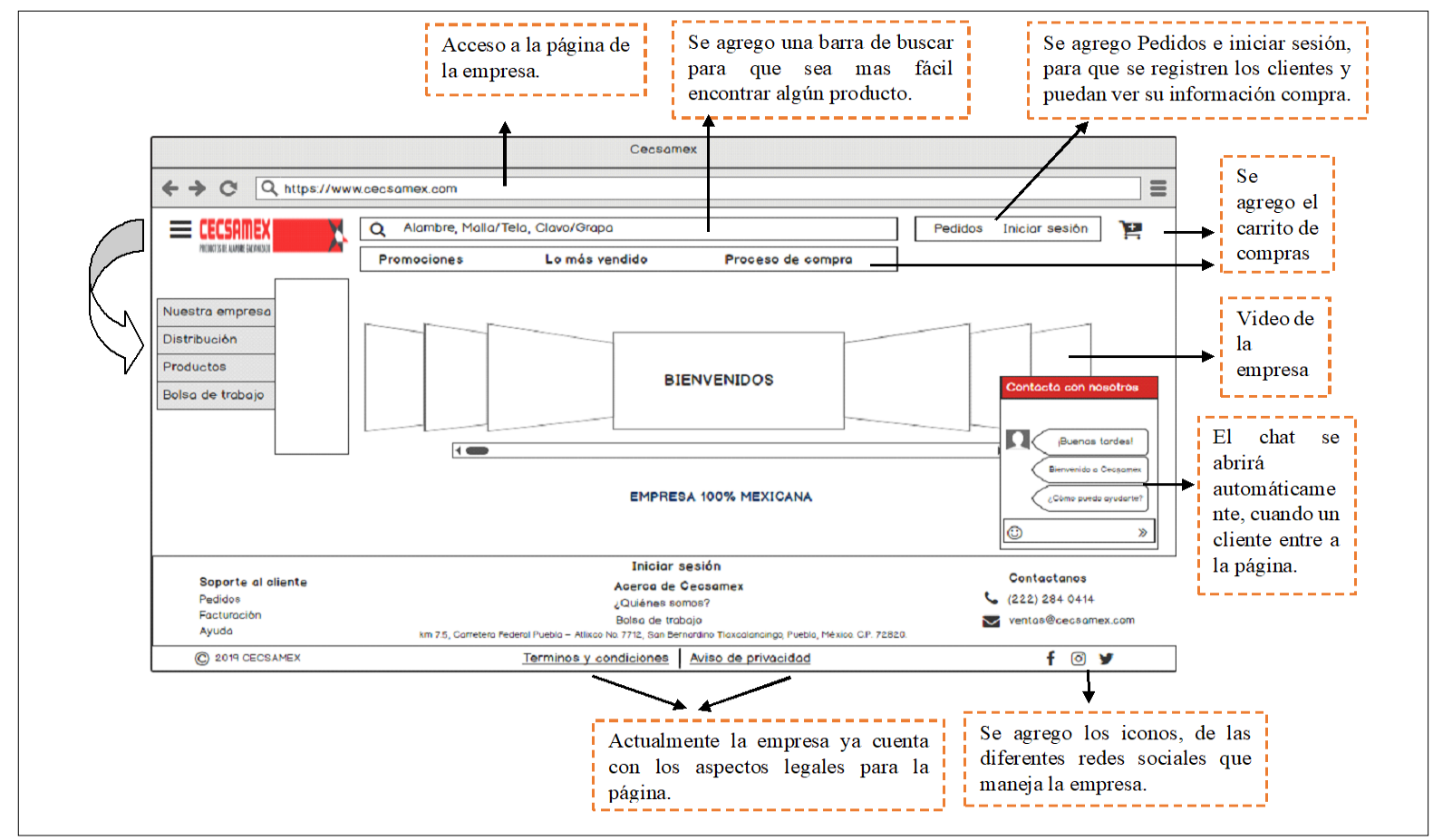

Figura 1. Diseño de modelo de comercio electrónico para CECSAMEX

\section{Colegio de Estudios Científicos y Tecnológicos (CECYTE)}

El Colegio de Estudios Científicos y Tecnológicos del Estado de Puebla, Plantel Cholula es un Organismo Público Descentralizado del Gobierno del Estado, fue inaugurado el 19 de mayo de 2012, el Plantel ofrece a los egresados de educación secundaria opciones de preparación múltiple propedéutica, mediante planes y programas, estudio que se fundamentan en el desarrollo de competencias genéricas, disciplinares y Profesionales, para que al término de su preparación reciban certificado de Bachillerato y una Acreditación en tecnologías Microsof.

En el caso del CECYTE, el proyecto fue realizado por la alumna Araceli Báez del $11^{\circ}$ cuatrimestre de ingeniería, durante el periodo enero-abril 2020, para hacer frente a la pandemia, con la finalidad de mantener las relaciones entre los diferentes segmentos a los que se dirige la institución, se propuso un plan de estrategias de mercadotecnia y relaciones públicas, que lleve a mejorar los procesos de difusión y promoción de la institución.

\section{Generación de estrategias}

Para analizar el ambiente institucional, se empleó la matriz FODA, en donde a partir de los resultados arrojados se identificaron los factores que dieron la pauta para el diseño de las estrategias, encontrándose que:

- Existe poca promoción del plantel en la región de influencia 
- No se cuenta con presupuesto para hacer promoción y difusión

- No existe material de identidad institucional

- Falta de medios propios para hacer el trabajo de difusión

- Planteles en la región que están posicionados entre la población

Para complementar el proyecto se identificó el nivel de posicionamiento que la institución ocupan en la región donde se ubica, por medio de un análisis de la competencia, la intención fue encontrar elementos que faciliten el diseño de las estrategias para el CEYTE.

\section{Matriz del perfil competitivo}

En la elaboración de la matriz de competencia, se tomaron como referencia los principales factores que intervienen en el proceso educativo en lo que los y las estudiantes, se ven inmersos. Para la valoración se les asignó una importancia porcentual y se realizó la evaluación de los principales competidores del Cecyte Cholula en la región, son: el Celma y el Cetis 67 (ver Figura 2).

\begin{tabular}{|c|c|c|c|c|c|c|c|}
\hline \multicolumn{8}{|l|}{ CECYTE CHOLULA } \\
\hline \multicolumn{2}{|c|}{ Factores y ponderación } & \multicolumn{6}{|c|}{ COMPETIDORES } \\
\hline \multirow{2}{*}{ Factores críticos para el éxito } & \multirow{2}{*}{ Importancia factor en \% } & \multicolumn{2}{|c|}{ CECYTE CHOLULA } & \multicolumn{2}{|c|}{ Celma } & \multicolumn{2}{|c|}{ Cetis 67} \\
\hline & & Puntos & Valor & Puntos & Valor & Puntos & Valor \\
\hline Oferta academica & $15.00 \%$ & 5.0 & 0.8 & 3.0 & 0.5 & 5.0 & 0.8 \\
\hline Promoción del servicio & $10.00 \%$ & 3.0 & 0.3 & 4.0 & 0.4 & 4.0 & 0.4 \\
\hline Calidad en el servicio & $15.00 \%$ & 4.0 & 0.6 & 4.0 & 0.6 & 4.0 & 0.6 \\
\hline Alianzas estrategicas & $15.00 \%$ & 5.0 & 0.8 & 4.0 & 0.6 & 4.0 & 0.6 \\
\hline Presencia en red social & $5.00 \%$ & 4.0 & 0.2 & 5.0 & 0.3 & 4.0 & 0.2 \\
\hline Personal docente y adm & $10.00 \%$ & 4.0 & 0.4 & 4.0 & 0.4 & 4.0 & 0.4 \\
\hline Infraestructura & $5.00 \%$ & 4.0 & 0.2 & 4.0 & 0.2 & 5.0 & 0.3 \\
\hline Ubicación & $10.00 \%$ & 3.0 & 0.3 & 4.0 & 0.4 & 5.0 & 0.5 \\
\hline Actvs culturales y deport & $5.00 \%$ & 4.0 & 0.2 & 4.0 & 0.2 & 5.0 & 0.3 \\
\hline \multirow[t]{2}{*}{ Innovación educativa } & $10.00 \%$ & 4.0 & 0.4 & 4.0 & 0.4 & 3.0 & 0.3 \\
\hline & $100.00 \%$ & & 4.1 & & 3.9 & & 4.3 \\
\hline
\end{tabular}

Figura 2. Matriz de perfil competitivo

Los resultados arrojaron que, a nivel general, el CECYTE obtuvo una calificación de 4.1 y se ubica en segundo lugar en relación a la competencia, lo cual indica que cuenta con la mayoría de los factores críticos del proceso educativo considerado exitoso, sin embargo, el área de oportunidad que se identificó para mejorar el proceso fue en la promoción y difusión del servicio educativo.

Una vea realizados ambos análisis (FODA y de la competencia), se cuenta con información relevante que revela el área estratégica a trabajar, de esta forma se establecieron los objetivos estratégicos, para finalmente diseñar 
una propuesta que permita al CECYTE establecer un plan de acciones a seguir para promocionarse en los diferentes segmentos en los que se debe estar vigente a pesar de la Pandemia.

\section{Programación de objetivos estratégicos}

En esta programación se establecen los objetivos y las estrategias, así como los indicadores de seguimiento y las metas concretas que cada uno debe cumplir en determinado tiempo que deben tomarse en cuenta en la institución.

Tabla 2. Objetivos estratégicos 2020

\begin{tabular}{|c|c|c|c|}
\hline $\begin{array}{l}\text { Objetivo } \\
\text { estratégico }\end{array}$ & Estrategias & Líneas de acción & Responsables \\
\hline \multirow{5}{*}{$\begin{array}{l}\text { Diseñar el } \\
\text { plan de } \\
\text { publicidad y } \\
\text { relaciones } \\
\text { públicas }\end{array}$} & $\begin{array}{l}\text { Implementar un } \\
\text { modelo de CRM }\end{array}$ & $\begin{array}{l}\text { Creación de una } \\
\text { base de datos con } \\
\text { información } \\
\text { relevante de los } \\
\text { estudiantes }\end{array}$ & \multirow{5}{*}{$\begin{array}{l}\text { Departamento } \\
\text { de vinculación }\end{array}$} \\
\hline & $\begin{array}{l}\text { Elaborar un plan de } \\
\text { referidos }\end{array}$ & $\begin{array}{l}\text { Entrevistas por } \\
\text { medio de zoom, } \\
\text { teams o meet, con } \\
\text { los alumnos y } \\
\text { padres de familia }\end{array}$ & \\
\hline & Crear publicidad nativa & $\begin{array}{c}\text { Difusión de } \\
\text { publicidad en } \\
\text { exteriores en } \\
\text { puntos } \\
\text { estratégicos como } \\
\text { paredes muros y } \\
\text { bardas. }\end{array}$ & \\
\hline & $\begin{array}{c}\text { Incorporar el marketing } \\
\text { directo, dirigido a } \\
\text { padres de familia }\end{array}$ & $\begin{array}{l}\text { Uso de mailing } \\
\text { como medio de } \\
\text { difusión con los } \\
\text { padres de familia. }\end{array}$ & \\
\hline & $\begin{array}{l}\text { Crear vínculos con } \\
\text { otros sectores } \\
\text { diferentes al giro } \\
\text { educativo }\end{array}$ & $\begin{array}{c}\text { Asistencia a } \\
\text { eventos como } \\
\text { congresos, foros y } \\
\text { expos. }\end{array}$ & \\
\hline
\end{tabular}

\section{Elaboración del Plan de actuación para el CECYTE}

La parte final fue la elaboración del plan actuación en donde se indican el área responsable, los objetivos, de qué manera se actuará, las situaciones iniciales y el resultado que se espera obtener, la duración del mismo y los indicadores para identificar el avance del mismo. 
Tabla 3. Plan de actuación

\begin{tabular}{|c|c|c|c|c|c|}
\hline Objetivo & $\begin{array}{c}\text { Líneas de } \\
\text { acción }\end{array}$ & $\begin{array}{c}\text { Situación } \\
\text { inicial }\end{array}$ & $\begin{array}{c}\text { Indicadore } \\
\mathrm{s}\end{array}$ & $\begin{array}{c}\text { Realizació } \\
\text { n en días }\end{array}$ & $\begin{array}{c}\text { Resultado } \\
\text { esperado }\end{array}$ \\
\hline \multirow{5}{*}{$\begin{array}{l}\text { Diseñar el } \\
\text { plan de } \\
\text { publicida } \\
\text { d y } \\
\text { relaciones } \\
\text { públicas }\end{array}$} & $\begin{array}{c}\text { Creación } \\
\text { de una base } \\
\text { de datos } \\
\text { con } \\
\text { informació } \\
\text { n relevante } \\
\text { de los } \\
\text { estudiantes }\end{array}$ & $\begin{array}{c}\text { Clasificación } \\
\text { de los } \\
\text { estudiantes }\end{array}$ & $\begin{array}{c}\text { Aumento de } \\
\text { la } \\
\text { matrícula, } \\
\text { formula: } \\
100 \% \text { de } \\
\text { alumnos } \\
\text { que } \\
\text { ingresan al } \\
\text { CECYTE / } \\
\text { Total de } \\
\text { alumnos } \\
\text { interesados }\end{array}$ & 30 días & $\begin{array}{l}\text { Dirección para } \\
\text { gestionar de } \\
\text { forma sencilla } \\
\text { las campañas } \\
\text { para la } \\
\text { captación de } \\
\text { alumnos }\end{array}$ \\
\hline & $\begin{array}{c}\text { Entrevistas } \\
\text { por medio } \\
\text { de zoom, } \\
\text { teams o } \\
\text { meet, con } \\
\text { los } \\
\text { alumnos y } \\
\text { padres de } \\
\text { familia }\end{array}$ & $\begin{array}{l}\text { Difusión en } \\
\text { redes } \\
\text { sociales }\end{array}$ & \multirow{3}{*}{$\begin{array}{l}\text { Alumnos } \\
\text { que } \\
\text { ingresan } \\
\text { por medio } \\
\text { de } \\
\text { promoción } \\
\text { Formula: } \\
100 \% \text { que } \\
\text { llegan por } \\
\text { pro y dif / } \\
\text { total de } \\
\text { alumnos }\end{array}$} & 6 días & \multirow[t]{2}{*}{$\begin{array}{c}\text { Captación de } \\
\text { mercado para el } \\
\text { aumento de la } \\
\text { matrícula }\end{array}$} \\
\hline & $\begin{array}{c}\text { Difusión de } \\
\text { publicidad } \\
\text { en } \\
\text { exteriores } \\
\text { en puntos } \\
\text { estratégicos } \\
\text { como } \\
\text { paredes } \\
\text { muros y } \\
\text { bardas } \\
\end{array}$ & $\begin{array}{l}\text { Identificació } \\
\text { n de zonas } \\
\text { aledañas de } \\
\text { la región }\end{array}$ & & 30 días & \\
\hline & $\begin{array}{l}\text { Uso de } \\
\text { mailing } \\
\text { como } \\
\text { medio de } \\
\text { difusión } \\
\text { con los } \\
\text { padres de } \\
\text { familia. }\end{array}$ & $\begin{array}{l}\text { Difusión con } \\
\text { amistades } \\
\text { para } \\
\text { promoción } \\
\text { de la } \\
\text { institución de } \\
\text { boca en boca }\end{array}$ & & 15 días & $\begin{array}{c}\text { Posicionamient } \\
\text { o del plantel en } \\
\text { la región }\end{array}$ \\
\hline & $\begin{array}{l}\text { Asistencia } \\
\text { a eventos } \\
\text { como } \\
\text { congresos, } \\
\text { foros y } \\
\text { expos }\end{array}$ & $\begin{array}{l}\text { Invitaciones } \\
\text { recibidas }\end{array}$ & $\begin{array}{l}\text { Total de } \\
\text { eventos en } \\
\text { los que } \\
\text { participa la } \\
\text { institución }\end{array}$ & 30 días & $\begin{array}{l}\text { Promoción de } \\
\text { la imagen y } \\
\text { oferta educativa } \\
\text { de la Institución }\end{array}$ \\
\hline
\end{tabular}




\section{PC IXOYE}

La empresa abrió operaciones en el año 2001, dedicada a la comercialización de productos electrónicos, se encuentra ubicada en la población de San Martín Texmelucan, para la realización de las propuestas para esta empresa colaboraron dos alumnos que cursaban el $6^{\circ}$ cuatrimestre de la carrera de TSU en DNM, Gerardo Valencia y Alejandro Ortiz, durante su estadía-practica en el periodo mayo-agosto 2020. La empresa PC IXOYE se considera pequeña y venía desarrollando sus actividades mercantiles de forma presencial, sin embargo, con la situación específica que atraviesa el mundo por la pandemia de Covid-19, y debido a que la recomendación de las autoridades sanitarias en México fue quedarse en casa sin exponerse a salir y realizar las actividades cotidianas con normalidad, la empresa vislumbró como área de oportunidad de incursionar en un nuevo formato para promover las ventas.

Una de la herramientas que se utilizan para diseñar las estrategias de forma correcta es lo que se denomina como, mezcla de mercadotecnia, marketing Mix o las 4P, que "consiste en la combinación de las variables controlables que un empresario en particular ofrece a los consumidores" (Fischer \& Espejo, 2004, p.7), y de acuerdo con los autores las principales son: Producto, Precio, Plaza y Promoción, se toman como base teórica para el desarrollo de las propuestas a la empresa.

El punto de partida fue por medio de un diagnóstico para conocer la situación en la que se encontraba la empresa, que debido al confinamiento físicamente se encontraba cerrada, por lo que una vez que se entrevistó a los propietarios, y con la información recopilada del diagnóstico, dado que la empresa no quería que se detuvieran sus operaciones, se identificaron los aspectos sobre los cuáles había que poner manos a la obra.

Diversos autores como Fischer y Espejo (2004), y Kottler (2000), se considera que, de las $4 \mathrm{P}$, la responsable de atraer a los consumidores e incentivarlos para que los consumidores adquieran el producto es la promoción, por lo que se desarrolló un mix en donde se combinaron diversos elementos para incentivar las ventas de la empresa, el caso de la empresa IP IXOE es peculiar debido a que se conjuntaron diversos elementos para para lograr la activación de las ventas en la empresa (ver la Figura 3). 


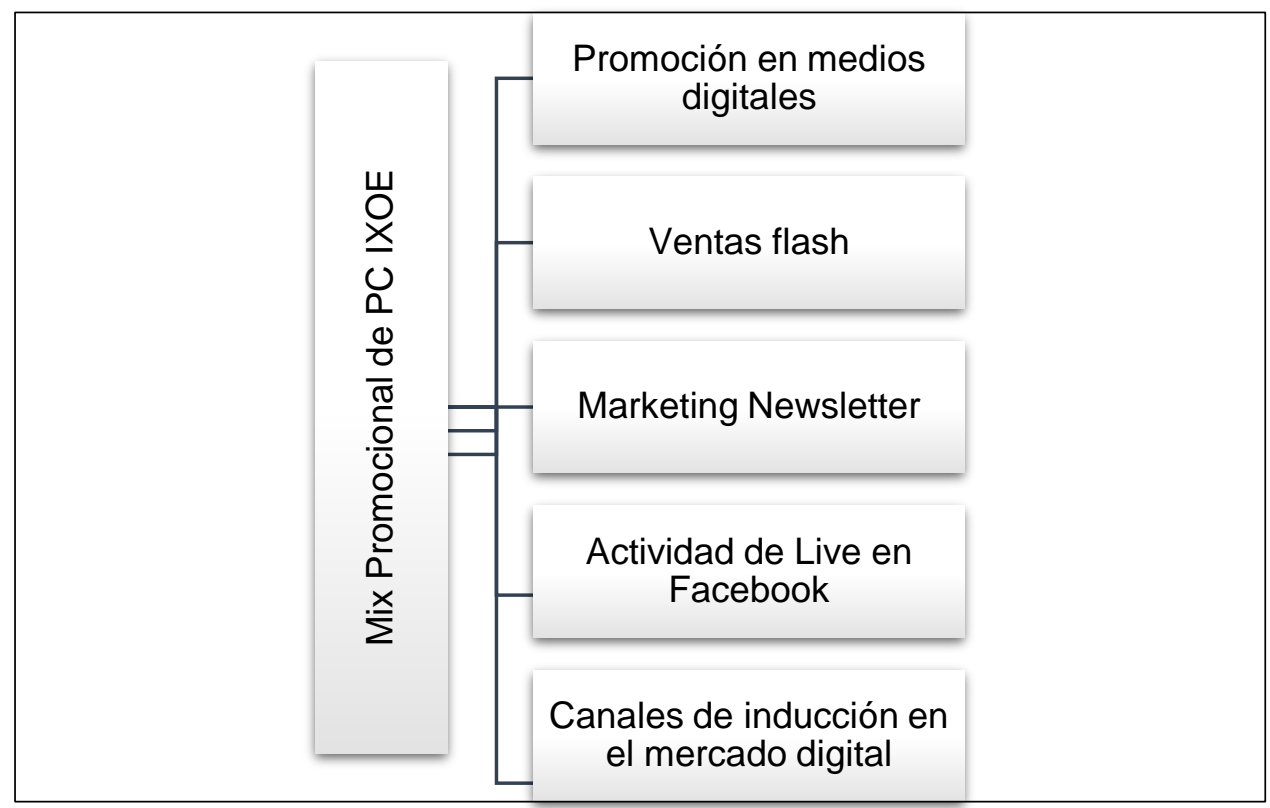

Figura 3. Estrategias del Mix promocional de PC IXOYE

Una vez elegidas las estrategias para la empresa se procedió a la implementación de las mismas, se describe como se realizó la implementación de cada una de ellas en la empresa. Es importante mencionar que a pesar de que la imagen corporativa no forma del proyecto, como resultado del diagnóstico se observó que la empresa no contaba con una imagen que le diera identidad propia, por lo que además de las estrategias, se diseñó un logotipo con la idea de que la empresa logre identificación y diferenciación en el mercado.

\section{Creación del logo para la empresa}

En el diseño del imagotipo se tomó en cuenta la las descripción que proporcionaron acerca del nombre de la empresa, IXOYE es una composición de ,"I" (Jesús) "X" (Cristo) “O” (Dios) "Y" (Hijo) y "E" (Salvador), entonces se tomó como representación al pez que acompaña a la palabra IXOYE, por ende el nombre está implícito en el imagotipo, la tipografía se tomó como inspiración la fuente algerian antes del boceto en papel, las curvaturas del diseño buscan la fluidez del mismo ya para que todo fuera orgánico y llamativo. Los colores fueron elegidos en una paleta que generaban una combinación atractiva y que no saturaran de un color primario al imagotipo (ver Figura 4). 


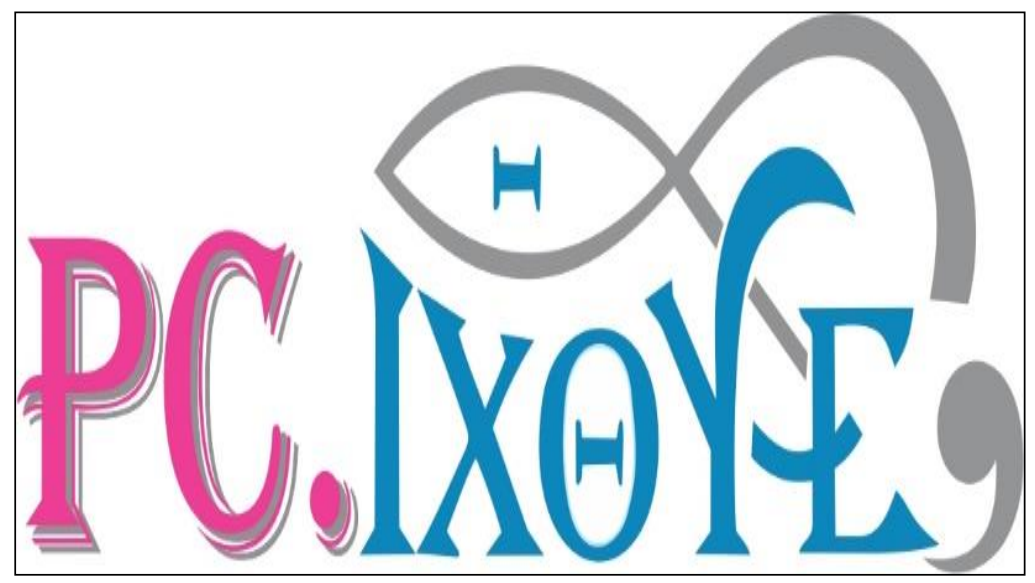

Figura 4. Logo de la empresa

\section{Promoción en medios digitales}

Se seleccionó como principal red social a Facebook debido, debido a que es una plataforma muy completa para interactuar con el usuario, además de que ofrece facilidades para la creación de una página específica para los negocio, se pretendía ingresar a grupos de compra al mayoreo en Facebook, pero los precios están muy por debajo de los de PC IXOyE, lo que se convirtió en una debilidad total, por otro lado, Facebook tiene un método en el cual te puede brindar publicidad que se ajusta al presupuesto del negocio para que las publicación llegue a más usuarios, la página se creó y está muy completa, puesto que ofrece al usuario información del negocio, contacto, visión, misión y una extensa gama de productos que se tienen en existencia.

\section{Ventas flash}

Son una estrategia que funcionó para el negocio, debido a que por medio de ellas se incentivó a los usuarios para realizar compras, consiste en subir un producto con un cierto límite de tiempo para que lo puedan adquirir, la forma en la que se desarrolló, fue colocar descuentos de un $15 \%$ en un artículo por un tiempo de 2 horas para motivar a los consumidores a su adquisición, de tal manera que cuando se publicó se vendieron 2 cámaras web, y a la vez, se consiguió que el usuario visitara la página Facebook de la tienda, por lo que fueron un gancho para que además los interesados preguntaran por la existencia de algunos artículos extras, que se fueron vendieron a precio lista. 


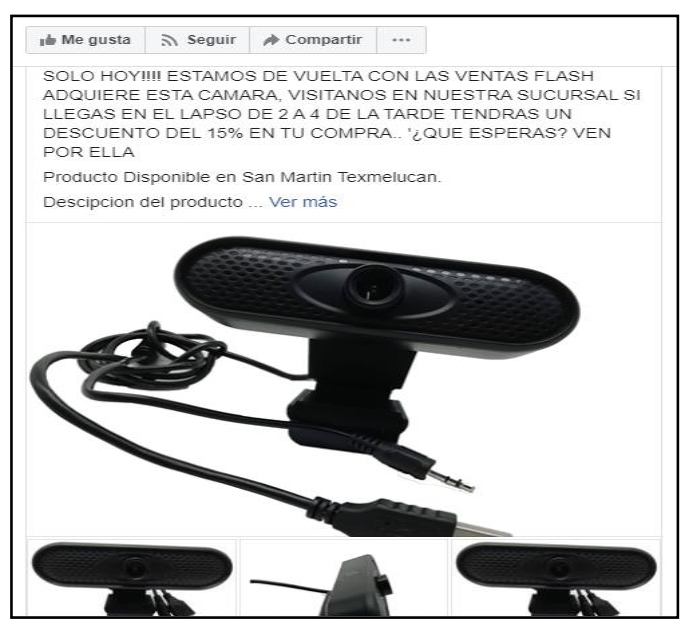

Figura 5. Ejemplo de ventas flash

\section{Marketing Newsletter}

Se utilizó una herramienta del marketing que ayudó tanto a la página, web como a la página de Facebook, que consistió en el diseñó de un pequeño formato llamado newsletter, donde se exponen el número de contacto, novedades y promociones que se publicaron semanalmente en la página de Facebook, la estrategia se desarrolló con el objetivo de atraer nuevos prospectos, y mantener información de las existencias y promociones que la tienda ofrece, por lo que, el formato es realmente sencillo pero eficaz. El formato se publica semanalmente, de preferencia debe ir acompañado de una promoción de ventas como descuento, o rebajas, ya que existen artículos que al ser devueltos o por tener algún defecto de menor, son los que se convirtieron en promocionables, cabe mencionar que una vez que se aplicó esto las visitas incrementaron en un 5\% de audiencia a la página, es decir, que comenzó a tener seguidores.

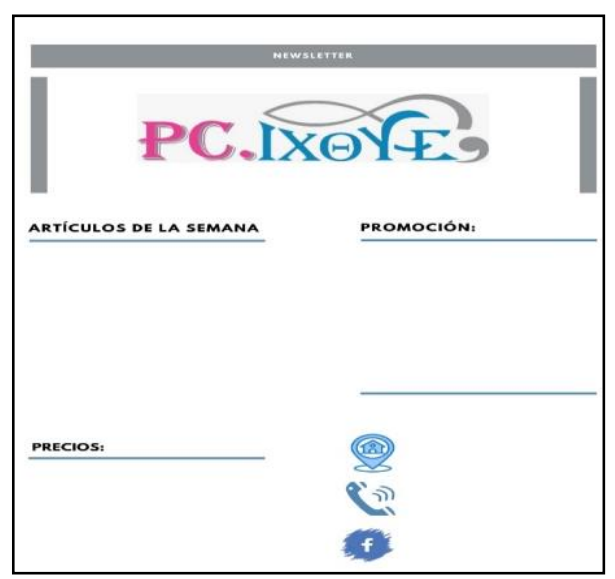

Figura 6. Ejemplo de Newsletter 


\section{Actividad de Live en Facebook}

Una herramienta que se tiene a alcance, que tuvo gran impacto, fue la realización de las transmisiones en vivo de los productos que se tenían en existencia en la tienda, la reacción de los usuarios fue positiva, en tiempo real comentaban sus dudas, como respuesta se logró llegar a más prospectos, la primera transmisión se llevó acabo el 5 de junio del año 2020, en ese momento solo se tenían 45 seguidores en la página, de los cuales solo 10 vieron la trasmisión, y 3 de los 10 comentaron en vivo que se los productos de estaban agotando, se programa una transmisión más el 12 de junio mismo año, cabe mencionar que se modificaron algunos aspecto como:

- La tonalidad de voz con la que realizamos la presentación del producto

- Introducción de dinámicas para que participara la comunidad virtual

- Invitación para que una vez concluida la pandemia se conozcan las Instalaciones

Cuando se mencionaron y aplicaron estas tres acciones, se unió un mayor número de personas a la transmisión, debido a que los productos más mostrados fueron la cámara web, que eran más difícil encontrarlas en el mercado, se convirtieron en un producto con alta demanda, especialmente entre la comunidad académica, derivado de que las clases eran en línea, una tarea de vital importancia consistió en identificar los productos que se convertían en una necesidad ante la pandemia por el COVID-19.

\section{Canales de inducción en el mercado digital}

Se identificó como el canal principal para la venta de productos por internet, con el cual la empresa comenzó a incursionar en el mercado online, la plataforma de venta argentina mercado libre, se incluyó un apartado de información y dudas en cuanto a los productos, y al mismo tiempo una gran gama para venta, en la actualidad el perfil de PC IXOYE, cuenta con 48 productos listos en la plataforma para venta inmediata, con fotos de calidad tomadas con cámara profesional en un mini estudio fotográfico portátil, con luz y fondo blanco, al inicio el perfil de la empresa solo se tenían 2 productos en venta, al mismo tiempo, se ganó en mercado libre puntos derivados de la popularidad por las ventas, y se obtuvo la insignia de vendedor confiable, lo que ayuda mucho si un usuario nuevo quiere comprar con la empresa.

\section{Discusión}

La pandemia ocasionada por el COVID-19, tomó por sorpresa a los negocios, especialmente a todos aquellos que venían operando de forma tradicional, en un lugar establecido donde físicamente el cliente compra y existe alguien que explica, atiende y sin mayor complicación se resuelven dudas o ejecuta la venta. Además, por decirlo de alguna forma, dejaban fuera o desconocían las herramientas y estrategias de la mercadotecnia para la 
operación de su negocio, entonces, es evidente que al igual que los casos expuestos en este trabajo, un gran número de medianas y pequeñas empresas o instituciones ya sea del sector público o privado, en México tienen claros cuáles son sus segmentos de mercado, porque se establecen en un lugar, y logran posicionamiento por el simple hecho del paso del tiempo.

Pero, lo que nadie imaginaba, mucho menos empresas e instituciones era como enfrentarse a una situación inédita, primero por la incertidumbre del arribo de un virus desconocido, seguido de un cierre total de todos los espacios físicos, para finalmente llegar al confinamiento, que si bien, se creyó que solo era cuestión de esperar algunos días para reincorpóranos a las actividades cotidianas, al transcurrir los días sucedió lo contario, aún al momento de cerrar estas líneas la pausa continúa. A medida en que los días se prolongaban, se inició la búsqueda de opciones para hacer frente a los problemas que se avecinaban para todos los sectores de la sociedad, comercio, educación, agricultura, turismo, industria por citar solo algunos, se comenzaron a padecer los estragos de la pandemia ocasionada por el COVID-19.

A raíz de la crisis por la que se atraviesa, que cada vez evidencia fuertes daños en las pequeñas y medianas industrias del país, surgió la necesidad de ir en busca nuevas oportunidades para seguir subsistiendo en el mercado. Había llegado el momento inevitable para la realización de los cambios, el reto era como lograrlo, en este caso, se apostó por apoyarse en todos los recursos que ofrece la mercadotecnia a las empresas. Se observó que la disposición que tuvieron las empresas para adaptabilidad, y transformación, realizada a través de la implementación de distintas herramientas y estrategias durante la pandemia, son elementos clave y asertivos para la reinvención de las PyMEs.

\section{Conclusiones}

La mercadotecnia ofrece un abanico de posibilidades para que las Pymes, pero también a otro tipo de instituciones sin fines de lucro, y que a pesar de que se presente alguna contingencia como es el caso de la pandemia por el COVID-19, además de que continúen también les permita seguir creciendo en el mercado, el tipo de herramienta o estrategia que se diseño va en función de que se analicen las necesidades particulares de cada negocio.

Ahora bien, una manera de identificar la herramienta y estrategia que se requiere en la empresa, es antes de todo se debe obtener información acerca de la empresa, lo cual puede hacerse por medio de una matriz FODA, entrevistas a propietarios y trabajadores, aplicación de un diagnóstico, o cualquier otro medio que revele lo que está sucediendo con la empresa, entonces, es a partir de los resultados que se obtengan que se cuenta con elementos que muestren el área de oportunidad para el diseño e implementación de una estrategia. 
A pesar de que en estos momentos el problema principal para todos es la pandemia, la realidad fue que, en el caso de los negocios, era necesario transformarse, por lo que en los casos revisados puede apreciarse, que en la empresa CECSAMEX se cambió de una página Web al comercio electrónico, es decir se trabajó en un modelo para que la empresa mantenga sus ventas por internet, cabe mencionar que se desarrolló todo el modelo de comercio electrónico, sin embargo, la puesta en marcha queda a cargo de la empresa, así como la evaluación para medir la efectividad.

EL segundo caso corresponde a una institución de educación media superior, se comprobó que la mercadotecnia no tiene por qué ser limitativa, por medio de una matriz FODA se identificó el punto crítico, se procedió a elaborar un plan de acción para la institución, donde se incluyeron, objetivo, estrategia, líneas de acción, tiempo y posibles resultados, que su aplicación se ha realizado de forma paulatina.

En la última de las empresas PC IXOYE, fue donde los propietarios mostraron disipación total, por lo que una vez hecha la entrevista, y observar como funcionada la empresa, se tuvieron elementos para el diseñó del mix promocional, puede decirse que esta empresa fue la única de las tres en donde se llegó a la implementación de estrategias, se obtuvieron resultados que son evaluables en relación a la efectividad de la estrategia.

Es importante dejar en claro que, de lo realizado en cada una de las empresas, solo en PC IXOYE se llegó a la implementación, las estrategias diseñadas fueron muy diversas, las hay desde aquellas que buscan incrementar la ventas aplicando promociones, descuentos temporales, uso de redes sociales, mercado libre; pero también las que hicieron uso de la publicidad en medios digitales, mailing, con la finalidad de posicionarse en la región y atraer un mayor número de población a la institución, pero también consideró el comercio electrónico.

En el caso de la empresa CECSAMEX y el CECYTE el trabajo realizado quedó en propuestas, sin embargo en las tres organizaciones, se incorporaron herramientas que por primera vez se utilizaban en cada una de ellas, es decir, que fue necesario cambiar el paradigma bajo la cual se entendió un nuevo esquema de trabajo, situación que las llevo a reinventarse, y que definitivamente, aún con la nueva normalidad las PyMEs en este caso durante la pandemia pueden seguir operando, tal vez la clave para seguir vigentes consiste en que tenga la capacidad para combinar los métodos tradicionales con las herramientas y estrategias que utilizan la tecnología que las pueda llevar a crecer en distintos segmentos de mercado.

\section{References:}

1. Carl, M., Joseph, H., \& Charles, 1. (2003). Fundamentos de Marketing. Pearson Educación: México. 
2. Fischer, L.., \& Espejo, A. (2007). Mercadotecnia. Mc Graw Hill: México.

3. Hair, MC. \& Daniel (2000). Marketing. Cengage Learning: Estados Unidos.

4. Herrera, J. \& Pintado, T. (2013). Imagen Corporativa. ESIC: España

5. Jiménez, E. (2004). Análisis de la investigación cuantitativa. Libro en pdf

en: http://pdfhumanidades.com/sites/default/files/apuntes/Jimenez\%20M arquez $\% 20-$

\%20M\%C3\%A9todos\%20c1\%C3\%A1sicos\%20an\%C3\%A1lisis\%20 cuanti\%20\%281\%29.pdf

6. Kotler, P. (2000). Fundamentos De Marketing. Pearson: México

7. Marciniak, R. (2013). Que es un plan estratégico. Recuperado de: https://renatamarciniak.wordpress.com/2013/01/07/que-es-un-planestrategico/

8. Palomares, R. (2009). Merchandinsing, teoría, práctica y estrategias. ESIC : España.

9. Segarra, J. (s/f). 5 pilares de marketing educativo. Recuperado de: https://www.tesubi.com/5-pilares-marketing-educativo/ 